\title{
Letrozole Plus 2 Different Gonadotropins Regimens for Intrauterine Insemination in Polycystic Ovary Patients: A Randomized Clinical Trial
}

\author{
Mina Naghi Jafarabadi ${ }^{1}$, Seyedeh Houra Mosavi Vahed ${ }^{2}$, Malihe Afiat $^{2 *}{ }^{\circledR}$, Zahra Ebrahimi $^{3}$, Zahra \\ Sadat Shiva ${ }^{4}$, Fedyeh Haghollahi ${ }^{1}$
}

\begin{abstract}
Objectives: The purpose of this study was to compare the effects of letrozole plus recombinant follicle stimulating hormone (R-FSH) and human menopausal gonadotropin (HMG) on ovarian stimulation in intrauterine insemination (IUI) cycle.

Materials and Methods: This randomized clinical trial was conducted on 130 women with polycystic ovary syndrome (PCOS) resistant to clomiphene and letrozole who were divided into A and B groups. Both groups received $5 \mathrm{mg}$ of letrozole daily from the third to seventh day of the cycle and then group A $(n=59)$ received 75 units of HMG intramuscularly per day and group B received 75 units of R-FSH subcutaneously per day. Follicular maturation was triggered with $250 \mu \mathrm{g}$ of recombinant human chorionic gonadotrophin (R-hCG) and IUI was performed 36 hours later. Main results under study were the number of matured follicles, endometrial thickness on the day of hCG injection, and the rate of chemical and clinical pregnancy.

Results: The 2 groups were not significantly different in demographic information. The rate of pregnancy was significantly higher in the letrozole + R-FSH group than in the letrozole + HMG group $(20 \%$ and $5.8 \%$ respectively, $P=0.04)$. There was no significant difference between the 2 groups in the number of mature follicles $(\leq 18 \mathrm{~mm})$ and endometrial thickness at the time of hCG injection $(P>0.05)$.

Conclusions: Letrozole plus R-FSH is a recommended regimen for ovarian stimulation in PCOS patients.

Keywords: HMG, Letrozole, Ovulation induction, Recombinant FSH, Polycystic ovary syndrome.
\end{abstract}

\section{Introduction}

Polycystic ovary syndrome (PCOS) is one of the most prevalent endocrine disorders in women of reproductive age that affects $5 \%$ to $10 \%$ of the women in the world and is the cause of $30 \%$ to $60 \%$ of ovulation disorders in infertile women (1). Finding a proper regimen for stimulation of ovulation in PCOS women is difficult (2). The most effective treatment primarily affects hypothalamichypophyseal-ovarian axis. As a selective estrogen receptor inhibitor, letrozole is suggested as the first choice in patients resistant to clomiphene (3). Letrozole inhibits the aromatase enzyme level in the body and reduces the conversion of androgen to estrogen (4). Aromatase inhibitor causes gonadotropin secretion and follicular growth by reducing blood estrogen negative feedback on the axis (3). Human menopausal gonadotropin (HMG) may cause multi-follicular growth or multiple gestation pregnancy and ovarian hyperstimulation syndrome (OHSS), and usually is used in combination with clomiphene or letrozole when ovulation does not happen with these medications (5-7). The question still remains about an effective treatment for ovulation induction in clomiphene-resistant patients. Research on the effects and safety of letrozole is still limited (2). A downside of gonadotropin includes multi-follicular growth and increased rate of cycle cancellation due to OHSS (8). Yun et al administered gonadotropin plus clomiphene or letrozole in intrauterine insemination (IUI) and showed that the efficacy was higher when combined with HMG (6). Research showed that combination treatment with letrozole significantly reduced the dosage of follicle stimulating hormone (FSH) required for the stimulation of ovulation in women with unknown causes of infertility $(6,9,10)$. However, there is limited research on these combination treatments in PCOS women. No research has been carried out so far to compare the effects and advantages of 2 different gonadotropins in combination with letrozole and the purpose of the present study is to compare these 2 methods in clomiphene-resistant PCOS women. Here, letrozole is administered in combination with gonadotropins for stimulation of ovulation in PCOS patients in order to investigate and compare the effects of 
letrozole + HMG and letrozole + R-FSH in stimulation of ovulation in women resistant to clomiphene.

\section{Materials and Methods}

This was a single-blind randomized study, which was performed at Milad hospital of Mashhad, Iran between February 2015 and February 2016.

PCOS patients who were resistant to clomiphene or letrozole in 2 previous cycles (no ovarian response to induction ovulation) and aged between 20 and 40 were included in the study. PCOS diagnosis was made according to the 2003 Rotterdam consensus criteria (7), i.e. to have 2 of the following three criteria: 1) ovulation dysfunction (sporadic ovulation or no ovulation) occurred after continuous monitoring for 2 or more natural cycles; 2). The results of the ultrasound showing polycystic ovaries; 3 ) hyperandrogenism, clinical or subclinical manifestations of androgen excess after ruling out hyperandrogenism caused by other diseases such as adrenal hyperplasia, Cushing syndrome and androgen-secreting tumors. This study has been approved by the ethics committee of the hospital, and informed consent was obtained from all patients. All cases were confirmed to have tubal patency (at least one tube) in hystrosalpingography and normal semen analysis. Patients with a history of ovarian surgery or complicated endometriosis, pelvic adhesion, liver, kidney, thyroid or prolactin dysfunction and smokers were excluded from the study. The PCOS patients were randomly divided into letrozole + R-FSH group $(n=59)$ and letrozole + HMG group $(n=59)$.

\section{Randomization}

All infertile women with PCOS who failed to ovulate with CC were individually counseled about the study and the protocols by a coordinator. After obtaining written consent they were allocated to one of 2 groups (A or B) via simple random sampling by the same coordinator. Group A $(\mathrm{n}=59)$ received $2.5 \mathrm{mg}$ of letrozole (Letrax, Aborayhan, Iran) twice daily for 5 days, starting on day 3 of the menstrual cycle and HMG 75 IU (Menopur, Ferring S.A., Madrid, Spain) was subcutaneously started on day 8 and was continued till the follicles size reached $15 \mathrm{~mm}$. Group B $(\mathrm{n}=59)$ received $2.5 \mathrm{mg}$ of letrozole (Letrax, Aborayhan, Iran) twice daily for 5 days, starting on day 3 of the menstrual cycle and r-FSH 75 IU (Gonal F, Serono, Madrid, Spain) was subcutaneously started on day 8 and continued till the follicles size reached 15 $\mathrm{mm}$. All patients were monitored for ovarian follicular development and endometrial thickness with transvaginal ultrasound on the 10th day of menstrual cycle. $250 \mu \mathrm{g}$ of recombinant human chorionic gonadotropin (hCG) (Ovitrelle, Serono, Madrid, Spain) was subcutaneously administered as a single dose when the average diameter of the leading follicles reached $\geq 18 \mathrm{~mm}$ (up 2 follicles). IUI was performed 36 hours later. Luteal phase was supported with the progesterone suppository (400 mg)
(Cyclogest, Actavis, Barnstaple, UK) daily for 15 days. Clinical pregnancy rate were the primary outcomes. Abortion and live birth were the secondary outcomes. Chemical pregnancy was confirmed through the analysis of serum $\beta$ hCG 14 days after IUI. Abortion was defined as loss of pregnancy before 20 weeks of gestation. Clinical pregnancy was defined as the presence of a gestational sac with cardiac activity as detected by transvaginal ultrasound.

\section{Statistical Analysis}

Data were analyzed using SPSS software version 16.0 (SPSS Inc., Chicago. IL). Student's $t$-test and chi-square test were used to analyze the obtained data. $P<0.05$ was considered statistically significant.

\section{Results}

A total of 130 consecutive patients (65 patients in group A and 65 patients in group B) meeting the inclusion criteria during the period from February 2015 to February 2016 were enrolled in this study. Six patients in group A and 6 patients in group B did not complete the treatment cycles and were excluded from the study (Figure 1). Demographic characteristics including age, duration of infertility, type of infertility and body mass index (BMI) are summarized in Table 1 and no significant difference was observed in 2 groups $(P>0.05)$. The number of follicles $\geq 18 \mathrm{~mm}$ at the time of hCG administration and the duration of follicular stimulation showed no difference in the 2 groups. The total dose of gonadotropins and endometrial thickness were similar in the 2 groups (Table 2). Pregnancy rates were different in the 2 groups $(8.5 \%$ and $22 \%$ in the group $\mathrm{A}$ and $\mathrm{B}$, respectively) $(P=0.04)$. There were not any significant differences in the abortion rate, twin pregnancies and live birth rate in 2 groups (Table 2). No cases of cycle cancelation or OHSS were reported in 2 groups.

\section{Discussion}

The strong point of our study is that it is the first randomized, prospective, single-blind clinical trial that administered the combination of letrozole with HMG

Table 1. Baseline of Characteristics in Patients

\begin{tabular}{lccc}
\hline Variables & $\begin{array}{c}\text { Group A } \\
(\mathbf{n}=59)\end{array}$ & $\begin{array}{c}\text { Group B } \\
(\mathbf{n}=59)\end{array}$ & P Value \\
\hline $\mathrm{Age}^{\mathrm{a}}(\mathrm{y})$, mean $\pm \mathrm{SD}$ & $28.25 \pm 4.64$ & $29.01 \pm 4.45$ & 0.59 \\
$\mathrm{BMI}^{\mathrm{a}}\left(\mathrm{kg} / \mathrm{m}^{2}\right)$, mean $\pm \mathrm{SD}$ & $23.14 \pm 3.22$ & $26.32 \pm 4.51$ & 0.54 \\
$\begin{array}{l}\text { Infertility duration } \\
\text { ( }(\mathrm{y}) \text {, mean }\end{array}$ & $4.73 \pm 3.34$ & $4.53 \pm 3.027$ & 0.54 \\
$\begin{array}{l}\text { Infertility type, No. }(\%)^{\mathrm{b}} \\
\quad\end{array}$ & & 0.09 \\
$\quad$ Primary & $48(81.4)$ & $40(67.8)$ & \\
$\quad$ Secondary & $11(18.6)$ & $19(32.2)$ & \\
\hline
\end{tabular}

Abbreviation: BMI, body mass index.

${ }^{\mathrm{a}} \boldsymbol{t}$ test; ${ }^{\mathrm{b}}$ Chi-square test. 


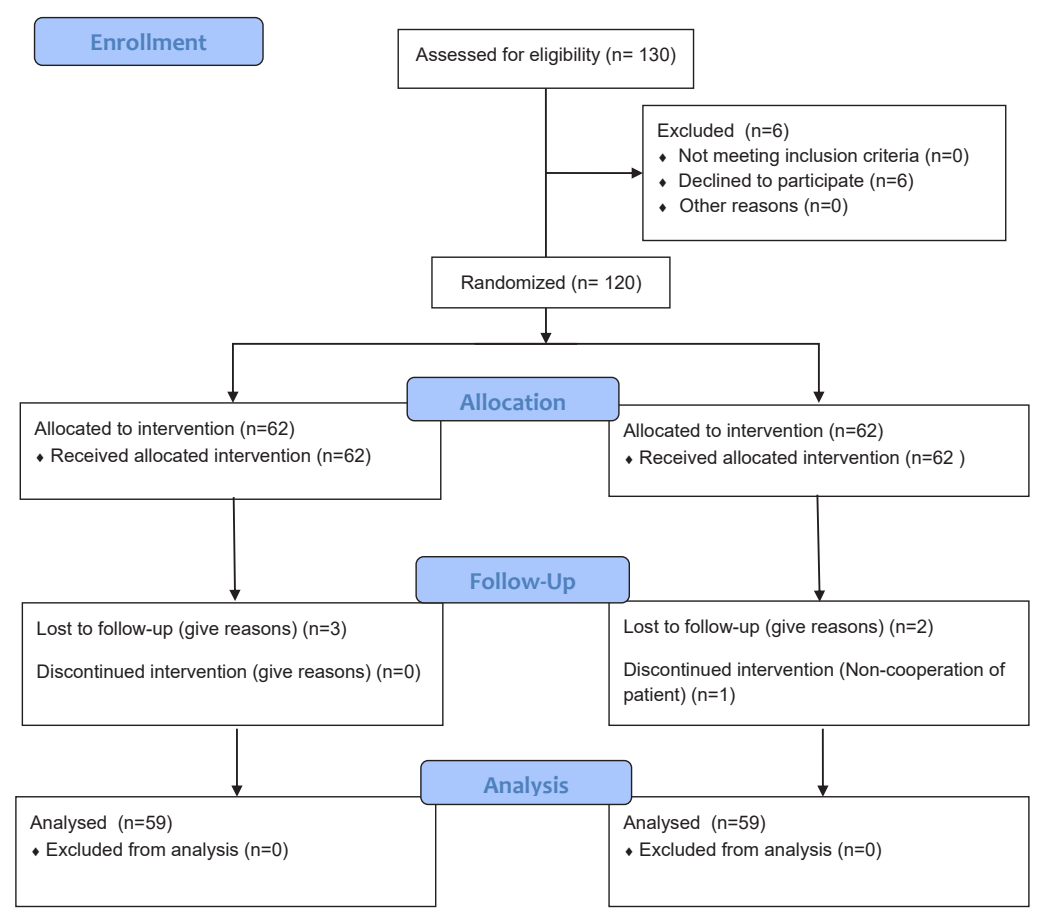

Figure 1. CONSORT Flow Diagram.

and with R-FSH for controlled ovarian stimulation in IUI cycle in PCOS patients. The rate of clinical and chemical pregnancy in the group B (letrozole $+\mathrm{R}-\mathrm{FSH}$ ) was higher than in the group A (letrozole + HMG).

Both letrozole and gonadotropin have been used alone in infertile women. Results suggest that aromatase inhibitor (letrozole) can be as effective as clomiphene and thus can be used as first-line treatment for ovarian stimulation. Letrozole seems to have a lower risk of multiple gestation (11) and OHSS (12).

PCOS patients who are resistant to clomiphene respond primarily to smaller dosages of gonadotropin. These women are also more sensitive to medication and the treatment range is narrow for them. If the dosage goes slightly higher, it will cause OHSS. Theoretically, recombinant FSH is more useful than HMG because it prevents excessive increase of LH in PCOS patients (11).

There is little research on the comparison of letrozole in combination with 2 different gonadotropins (HMG and R-FSH) in PCOS patients. The study of Yun et al on the combined administration of gonadotropins and clomiphene or letrozole in the IUI cycle showed that the

Table 2. Clinical Variables and Outcomes in Patients

\begin{tabular}{|c|c|c|c|}
\hline Variables & Group A ( $n=59)$ & Group B ( $n=59)$ & $P$ Value \\
\hline Number of Gonadotropin, ${ }^{a}$ mean \pm SD & $4.42 \pm 1.77$ & $4.23 \pm 1.60$ & 0.60 \\
\hline Endometrial thickness on day of $\mathrm{hCG}$ injection $(\mathrm{mm}),{ }^{a}$ mean $\pm \mathrm{SD}$ & $8.50 \pm 1.82$ & $8.08 \pm 1.65$ & 0.42 \\
\hline Follicular phase duration (day), ${ }^{a}$ mean \pm SD & $13.22 \pm 1.71$ & $12.93 \pm 2.25$ & 0.78 \\
\hline Mature follicle (mono follicle), ${ }^{\mathrm{b}}$ No. (\%) & $33(54.2)$ & $28(49.2)$ & 0.74 \\
\hline Mature follicles (2 follicles), ${ }^{\mathrm{b}}$ No. (\%) & $8(13.6)$ & $9(15.3)$ & 0.73 \\
\hline Chemical pregnancy, ${ }^{\mathrm{b}}$ No. (\%) & $5(8.5)$ & $13(22)$ & 0.04 \\
\hline Clinical pregnancy, ${ }^{\text {b }}$ No. (\%) & $5(8.5)$ & $13(22)$ & 0.04 \\
\hline Abortion rate, ${ }^{b}$ No. (\%) & $0(0.0)$ & $2(3.4)$ & 0.15 \\
\hline Live birth rate, ${ }^{\mathrm{b}}$ No. (\%) & $5(8.5)$ & $11(18.6)$ & 0.10 \\
\hline Twin pregnancy, ${ }^{\mathrm{b}}$ No. (\%) & $3(5.1)$ & $2(3.4)$ & 0.64 \\
\hline
\end{tabular}

Abbreviation: hCG, human chorionic gonadotrophin.

${ }^{a} t$ test; ${ }^{\mathrm{b}} \mathrm{Chi}$-square test. 
efficacy washigher when combined with HMG(6). Previous studies showed that treatment with letrozole reduced the dosage of HMG required and duration of treatment more effectively and significantly $(11,12)$. Torabizadeh and Mirzaian reported that treatment of PCOS patients with different ovulation induction methods such as FSH, HMG or the combination of these medications did not make any difference to pregnancy outcomes (13). Zhao et al argued that letrozole plus purified HMG was a safe and effective method for clomiphene-resistant patients (14).

Athwal et al showed that letrozole plus FSH was an effective treatment for stimulation of ovulation in infertile women (15). The present study showed that the rate of clinical and chemical pregnancy was higher in group $\mathrm{B}$ (letrozole + R-FSH) than in group A (letrozole + HMG). Because of the increase of LH in PCOS patients (16) and also the early increase of LH in plasma and follicular fluid, the growth of granulosa cells of antral follicles will decrease and follicular degeneration will rise (11). Therefore, administration of letrozole + R-FSH seems to be more effective than letrozole + HMG by inhibiting the early increase of endogen LH level. Research limitations include lack of LH level measurements before and after the intervention and small sample size that are recommended to be addressed in future research.

\section{Conclusions}

Administration of the combination of Letrozole and r-FSH seems to be a proper treatment in PCOS patients resistant to clomiphen citrate (CC) in comparison to many other therapeutic regimens including HMG or letrozole + HMG with no more prevalent side effects.

\section{Conflict of Interests}

The authors have no conflicting interests that might be perceived to influence the results and discussion reported in this paper.

\section{Ethical Issues}

This study was approved by the Ethics Committee of Mashhad University of Medical Sciences (Ethics No. IR.MUMS.REC.1394.311). All participants signed an informed consent for participation in this study. This was a single-blind randomized, prospective study registered in the IRCT website (identifier: IRCT201703153950N6) that was performed at Milad hospital of Mashhad, Iran between February 2015 and February 2016.

\section{Financial Support}

None.

\section{Acknowledgments}

This paper is a Medical thesis, which was approved by Mashhad University of Medical Sciences. We are grateful to the Infertility Center of Milad Hospital affiliated to Mashhad University of Medical Sciences and Vali-
Asr Reproductive Health Research Center of Tehran University of Medical Sciences for helping us.

\section{References}

1. Franks S. Polycystic Ovary Syndrome. N Engl J Med. 1995;333(13):853-861. doi:10.1056/nejm199509283331307

2. Chen Z, Zhang M, Qiao Y, Yang J. Effects of letrozole in combination with low-dose intramuscular injection of human menopausal gonadotropin on ovulation and pregnancy of 156 patients with polycystic ovary syndrome. Pak J Med Sci. 2016;32(6):1434-1438. doi:10.12669/ pjms.326.11391

3. Legro RS. Ovulation induction in polycystic ovary syndrome: Current options. Best Pract Res Clin Obstet Gynaecol. 2016;37:152-159. doi:10.1016/j.bpobgyn.2016.08.001

4. Casper RF, Mitwally MF. Use of the aromatase inhibitor letrozole for ovulation induction in women with polycystic ovarian syndrome. Clin Obstet Gynecol. 2011;54(4):685695. doi:10.1097/GRF.0b013e3182353d0f

5. Xi W, Liu S, Mao H, Yang Y, Xue X, Lu X. Use of letrozole and clomiphene citrate combined with gonadotropins in clomiphene-resistant infertile women with polycystic ovary syndrome: a prospective study. Drug Des Devel Ther. 2015;9:6001-6008. doi:10.2147/dddt.s83259

6. Yun $\mathrm{BH}$, Chon SJ, Park JH, et al. Minimal stimulation using gonadotropin combined with clomiphene citrate or letrozole for intrauterine insemination. Yonsei Med J. 2015;56(2):490-496. doi:10.3349/ymj.2015.56.2.490

7. Revelli A, Chiado A, Dalmasso P, et al. "Mild" vs. "long" protocol for controlled ovarian hyperstimulation in patients with expected poor ovarian responsiveness undergoing in vitro fertilization (IVF): a large prospective randomized trial. J Assist Reprod Genet. 2014;31(7):809815. doi:10.1007/s10815-014-0227-y

8. Nahuis MJ, Weiss NS, van der Veen F, et al. The M-OVIN study: does switching treatment to FSH and / or IUI lead to higher pregnancy rates in a subset of women with world health organization type II anovulation not conceiving after six ovulatory cycles with clomiphene citrate - a randomised controlled trial. BMC Womens Health. 2013;13:42. doi:10.1186/1472-6874-13-42

9. Healey S, Tan SL, Tulandi T, Biljan MM. Effects of letrozole on superovulation with gonadotropins in women undergoing intrauterine insemination. Fertil Steril. 2003;80(6):1325-1329.

10. Mitwally MF, Casper RF. Aromatase inhibition reduces gonadotrophin dose required for controlled ovarian stimulation in women with unexplained infertility. Hum Reprod. 2003;18(8):1588-1597. doi:10.1093/humrep/ $\operatorname{deg} 311$

11. Fritz MA, Speroff L. Clinical Gynecologic Endocrinology and Infertility. Lippincott Williams \& Wilkins; 2012: 13091314.

12. Kamath MS, Maheshwari A, Bhattacharya S, Lor KY, Gibreel A. Oral medications including clomiphene citrate or aromatase inhibitors with gonadotropins for controlled ovarian stimulation in women undergoing in vitro fertilisation. Cochrane Database Syst Rev. 2017;11:Cd008528. doi:10.1002/14651858.CD008528.pub3

13. Torabizadeh A, Mirzaian S. Comparison of the IVF outcome 
between three methods of induction ovulation in PCOS patients. The Iranian Journal of Obstetrics, Gynecology and Infertility. 2011;14(1):7-13. (Persian).

14. Zhao Y, Ruan X, Mueck AO. Letrozole combined with low dose highly purified HMG for ovulation induction in clomiphene citrate-resistant infertile Chinese women with polycystic ovary syndrome: a prospective study. Gynecol Endocrinol. 2017;33(6):462-466. doi:10.1080/09513590.20 17.1292241

15. Athwal A, Chakravorty R, Sur D, Saha R. Use of letrozole versus clomiphene citrate combined with gonadotropins for ovulation induction in infertile women with polycystic ovary syndrome: a pilot study. Int J Reprod Contracept Obstet Gynecol. 2017;6(9):4098-4101. doi:10.18203/23201770.ijrcog20174068

16. Piouka A, Farmakiotis D, Katsikis I, Macut D, Gerou S, Panidis D. Anti-Mullerian hormone levels reflect severity of PCOS but are negatively influenced by obesity: relationship with increased luteinizing hormone levels. Am J Physiol Endocrinol Metab. 2009;296(2):E238-243. doi:10.1152/ ajpendo. 90684.2008

(C) 2018 The Author (s); This is an open-access article distributed under the terms of the Creative Commons Attribution License (http://creativecommons.org/licenses/by/4.0), which permits unrestricted use, distribution, and reproduction in any medium, provided the original work is properly cited. 